\title{
Cils et kystes rénaux
}

Mélanie Paces-Fessy

> Les avancées en génomique et en bioinformatique, ainsi que la création d'organismes modèles, ont permis d'identifier de nombreux gènes associés à la formation de kystes rénaux. Même si ces gènes ne sont pas obligatoirement liés aux ciliopathies, de nombreuses connexions ont été identifiées entre les maladies kystiques du rein et les fonctions du cil primaire. En effet, les gènes associés aux kystes rénaux codent pour des protéines qui sont localisées au niveau du cil lui-même ou au niveau du corps basal, ou encore qui régulent l'expression et la localisation de protéines ciliaires. Le but de cet article est de décrire les multiples processus cellulaires qui, lorsqu'ils sont dérégulés, peuvent conduire à l'apparition de kystes rénaux: modifications de la prolifération ou de la polarité cellulaires, altérations des voies de signalisation impliquées dans le développement embryonnaire rénal. Afin d'éclairer le rôle du cil primaire dans la formation de kystes rénaux, je discuterai plusieurs études qui ont caractérisé la fonction des gènes ciliaires et du cil dans les reins de différents organismes modèles. <

\section{Ciliopathies et reins kystiques}

Les pathologies kystiques du rein représentent un groupe de maladies multigéniques et pour partie associées aux polykystoses autosomiques dominantes (autosomal dominant polycystic kidney disease, ADPKD), autosomiques récessives (autosomal recessive polycystic kidney disease, ARPKD), aux néphronophtises (NPHP) ou aux syndromes Bardet-Biedl (BBS) et Meckel-Joubert (MKSJBTS) (Tableau I) [70] $(\rightarrow)$.

$(\rightarrow)$ Voir la Synthèse de R. BachmannGagescu, page 1011 de ce numéro

Cet article fait partie du numéro thématique de médecine/sciences intitulé «Cils primaires et ciliopathies $\left(\mathrm{m} / \mathrm{s} \mathrm{n}^{\circ} 11\right.$, vol. 30 , novembre 2014).

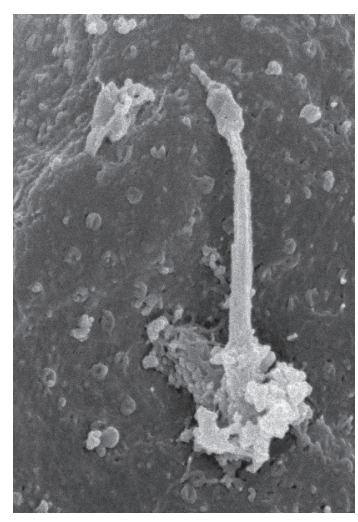

Sorbonne universités, université Paris 6 UPMC, UMR 7622 CNRS, Inserm U1156, IBPS (institut de biologie Paris Seine), laboratoire de biologie du développement, 9 , quai Saint-Bernard, 75005 Paris, France. melanie.paces-fessy@upmc.fr

Selon le type de pathologie, les patients développent, dès la naissance, dans l'enfance ou à l'âge adulte, une insuffisance rénale associée parfois à une augmentation de la taille des reins et à la présence de kystes rénaux bilatéraux de localisation variable [ 1 , 2]. À l'exception des cas de kystes isolés, les pathologies kystiques rénales sont majoritairement regroupées sous le terme de ciliopathies et sont fréquemment associées à des manifestations extrarénales, kystes des canaux biliaires et du pancréas chez les patients atteints de polykystose, anomalies du squelette, du cerveau ou de la rétine dans les autres ciliopathies $[70](\rightarrow)$.

$\rightarrow$ Voir la Synthèse de R. BachmannGagescu, page 1011 de ce numéro En dépit de l'hétérogénéité des maladies conduisant à la formation de kystes rénaux, la plupart des gènes qui sont impliqués interviennent dans la structure et/ou la fonction du cil primaire (Tableau I). Le cil primaire est un organite cellulaire localisé à la surface de presque toutes les cellules de vertébrés, et qui fonctionne

$(\rightarrow)$ Voir la Synthèse de C. Fort et P. Bastin, page 955 de ce numéro comme mécanosenseur ou intégrateur de plusieurs voies de signalisation [71] $(\rightarrow)$.

Ainsi, les protéines polycystine- 1 et -2 ( $P C 1, P C 2)$, impliquées dans les ADPKD, forment un canal calcique hétérodimérique, notamment localisé sur le cil, qui transmet une réponse calcique induite par le flux [3]. Les interactions entre PCl et PC2 sont essentielles à la fonction du canal. Ces deux protéines sont exprimées au cours du développement embryonnaire, lors de la formation du rein. Leur rôle y est encore mal caractérisé. Toutefois, PCl semblerait impliquée in vivo et in vitro dans l'arborescence des canaux collecteurs du rein, ainsi que dans l'élongation et la maturation des tubules [4]. La polykystose autosomique dominante résulterait d'une perturbation de l'activité du canal calcique, qui favoriserait l'initiation de la formation des kystes et leur prolifération, et ce à l'âge adulte chez les patients comme chez la souris $[5,6]$. 


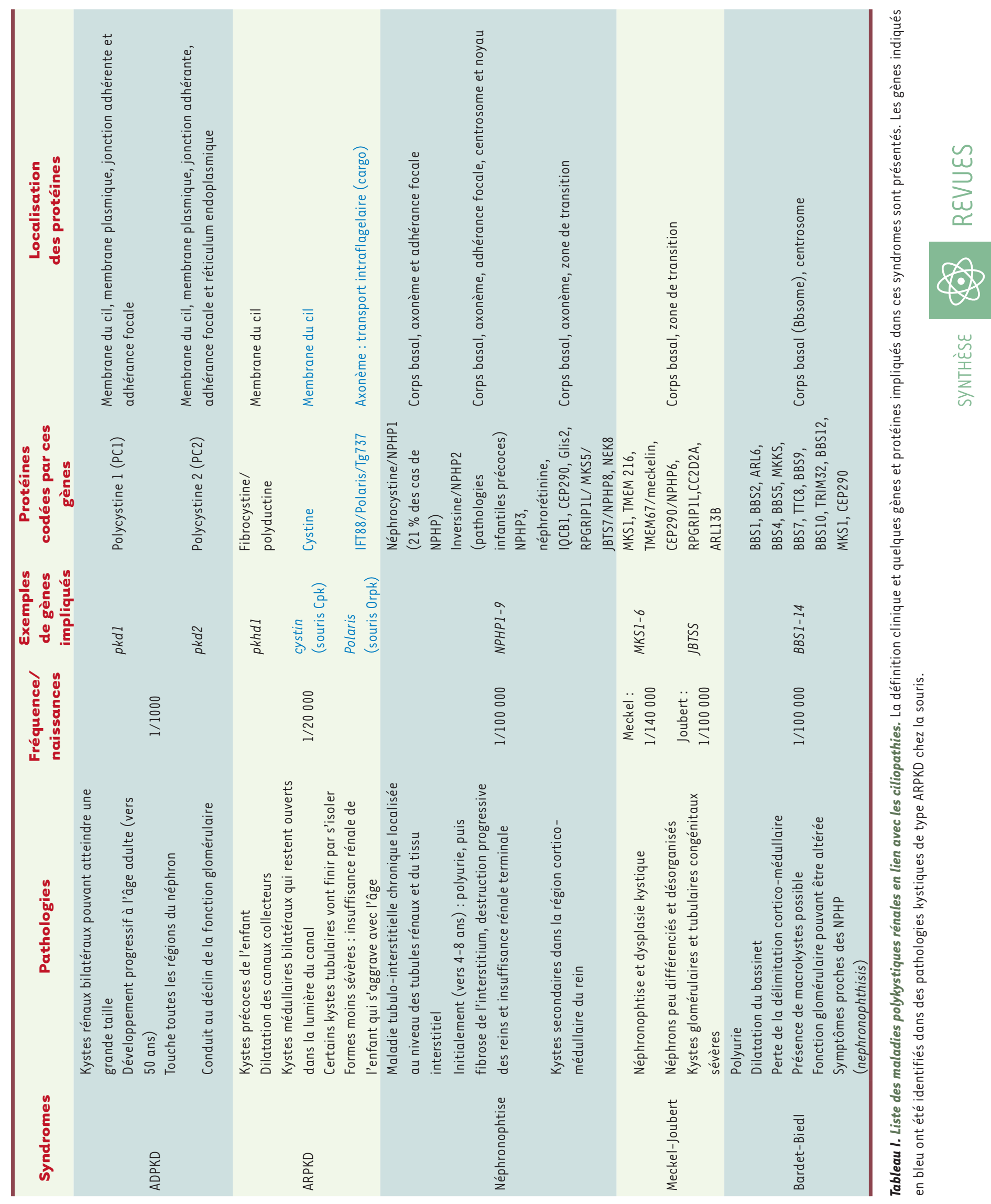


La fibrocystine, impliquée dans les polykystoses autosomiques récessives, est également associée aux cils. Elle interagit avec $\mathrm{PCl}$ et $P C 2$, et cette interaction, dépendante du moteur moléculaire kinésine-2, modulerait l'activité du canal calcique [7]. Les kystes des ARPKD touchent, à la différence de ceux des ADPKD, principalement les canaux collecteurs du rein et apparaissent très précocement chez l'enfant.

Les autres maladies récessives kystiques du rein ont des spectres cliniques chevauchants liés, soit à des mutations dans des gènes différents, soit à différentes mutations d'un seul gène, comme par exemple MKS5 (Meckel syndrome protein 5)/NPHP8 (nephronophthisis protein 8)/rpGripll (retinitis pigmentosa GTPase regulatorinteracting protein $1 L$ ). Les produits des gènes NPHP, MKS et BBS, localisés au niveau du corps basal, de la zone de transition ou de l'axonème du cil, contribuent à la formation de cet organite et à la transduction de signaux dépendants du cil, tels que les voies Wht et Hedgehog $(\mathrm{Hh})[71](\rightarrow)$.

In vitro, les cellules rénales modifiées de façon à modéliser les altérations des gènes NPHPI, $-3,-4,-6$, et -8 ou MKS1 expriment des défauts de polarité apicale et ont des cils plus courts, défectueux, voire absents [8-10]. Toutefois, in vivo, les résultats sont plus ambigus, puisque certaines des souris mutantes pour ces gènes n'expriment aucun phénotype rénal. Chez la souris Inv ${ }^{-/-}\left(\mathrm{Nphp}^{\mathrm{C}^{-/}}\right)$, la présence de kystes rénaux n'est d'ailleurs associée à aucune altération de la structure, de la longueur ou du mécanisme de flexion des cils primaires du rein, contrairement à l'effet de cette même mutation sur les cils nodaux $[11,12]$.

Enfin, les souris Orpk (Oak Ridge polycystic kidney) et Cpk (congenital polycystic kidney), porteuses de mutations des gènes codant respectivement pour les protéines IFT88 (intraflagellar transport protein 88) (Tg737/Polaris) et cystine, toutes deux localisées au niveau du cil [13], développent également des kystes rénaux embryonnaires. Les protéines IFT, impliquées dans le système de transport intraflagellaire dans le cil (complexe B) et hors du cil (complexe $A$ ), sont requis pour la ciliogenèse rénale [71] $(\rightarrow)$.

Ainsi, le lien entre cil primaire et kystes rénaux a-t-il été proposé pour la première fois à la suite de l'étude d'IFT88 [14]. Des études récentes montrent que des mutations dans les gènes codant toutes les protéines du complexe IFT-A, et certains de ceux codant les protéines du complexe IFT-B, sont responsables de différentes formes de ciliopathies associant maladie rénale kystique (NPHP) et défauts squelettiques $[15,16]$. Chez la souris, les mutations Ift20 (IFT-B), Ift140 (IFT-A), ainsi que celles qui touchent les moteurs moléculaires kinésine-2 (Kif3a [kinesin family member $3 A]$ et Kif3b) génèrent également des kystes rénaux se développant après la naissance [17, 18]. Toutefois, les IFT peuvent exercer des fonctions extraciliaires pouvant conduire à d'autres défauts rénaux de type glomérulopathie $[19,72](\rightarrow)$.

\section{Développement du métanéphros et formation de kystes}

Les reins sont des organes complexes formés de plusieurs types cellulaires très spécialisés organisés en canaux collecteurs et néphrons (unités de filtration du rein). Ces derniers sont constitués d'un glomérule et de différents segments tubulaires, dont la fonction dépend de l'organisation spatiale qui se met en place au cours du développement embryonnaire (Figure 1) [20]. Deux modes de formation des tubules surviennent simultanément dans le rein; une arborescence progressive, qui concerne l'organisation des canaux collecteurs, et la formation d'un épithélium via une transition mésenchyme-épithélium, qui préside à l'émergence des néphrons. Le rein est donc formé de plusieurs tubules épithéliaux, dont l'origine développementale et les processus de tubulogenèse diffèrent. Ces tubules peuvent tous être affectés par l'émergence de kystes. En effet, dans les maladies kystiques du rein, il semblerait que le contrôle strict de la longueur et du diamètre d'un tube, essentiel à sa fonction, soit perdu.

Dans les ADPKD, le kyste rénal se constitue comme une dilatation progressive des tubules rénaux. Ces dilatations se remplissent de liquide qui exerce une pression hydrostatique entraînant l'augmentation du diamètre de la lumière du tube, donc de la taille du kyste, et conduisant au détachement de celui-ci du tubule. Le kyste forme alors une structure remplie de liquide indépendante du néphron [21]. Le glomérule peut également s'élargir et former des kystes glomérulaires. À terme, l'apparition de ces kystes et leur progression conduisent à une insuffisance rénale terminale pour laquelle la dialyse ou la greffe sont les seules options thérapeutiques. Les kystes peuvent être observés au cours du développement embryonnaire dès $\varepsilon 15,5 \mathrm{chez}$ la souris, par exemple chez les mutants nuls Pkdl [22]. À ce stade, le rein embryonnaire devient fonctionnel et commence à filtrer le sang, ce qui renforce l'idée selon laquelle le flux jouerait un rôle dans la progression des kystes. Plusieurs études réalisées dans des modèles murins d'inactivation génique conditionnelle dans lesquels le gène étudié est inactivé à différents stades du développement ou en période postnatale, ont montré l'importance physiologique de la diminution de la prolifération cellulaire qui survient 12 à 14 jours après la naissance [23-26]. La prolifération peut toutefois être réactivée chez l'adulte par des lésions stimulant la régénération de tubules. Les kystes qui se déclenchent au-delà de ce stade de 14 jours - qui marque un changement du statut développemental du rein - ne sont plus que des microkystes de moindre sévérité 


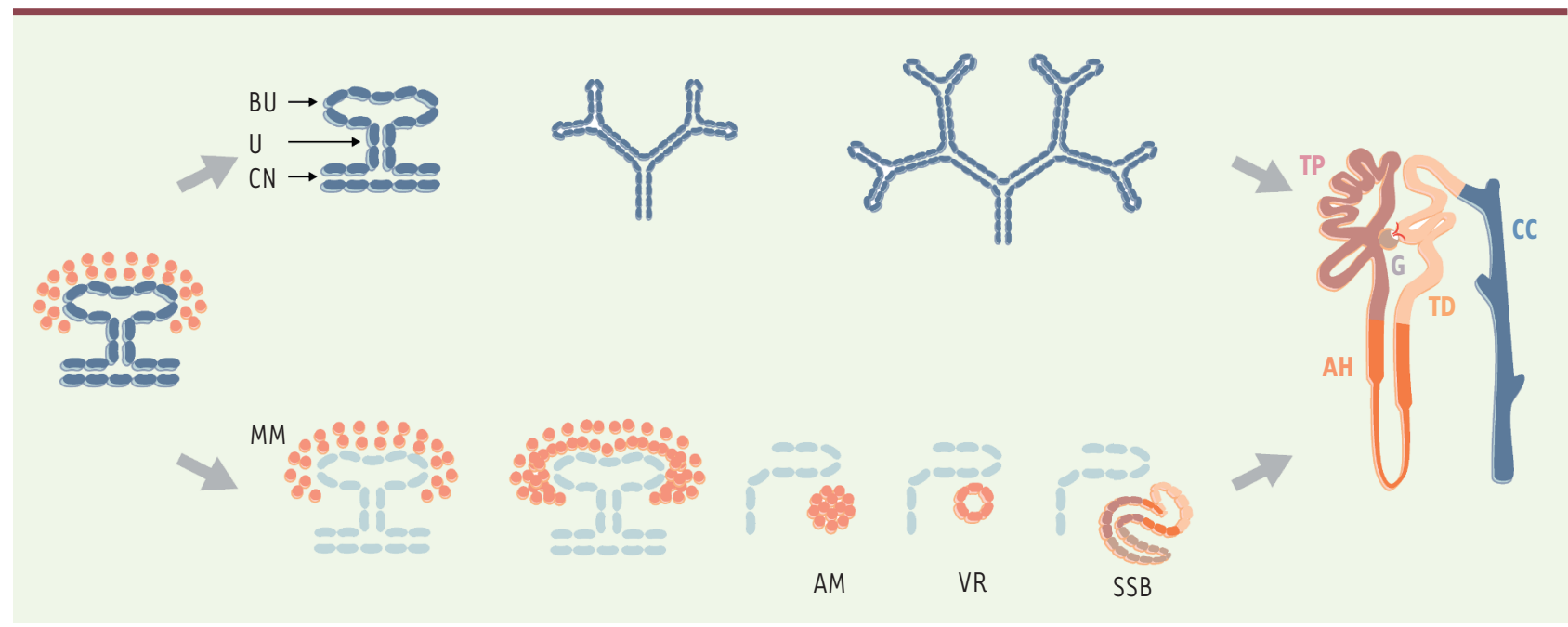

Figure 1. Le développement du rein fonctionnel. Le métanéphros commence par l'émergence du bourgeon urétéral (BU) dans la région caudale du canal néphrique (CN ou canal de Wolff) [20]. Lors de l'invasion du mésenchyme adjacent (MM), le BU tubulaire va réaliser plusieurs vagues de branchements dichotomiques pour aboutir à la formation d'un réseau tubulaire à l'origine du système de canaux collecteurs (CC) du rein. La tige du BU va, quant à elle, se différencier en uretère $(U)$, conduit qui achemine l'urine du rein vers la vessie. En parallèle, dans chaque branche dérivant du BU, les cellules de l'extrémité vont induire la formation d'agrégats mésenchymateux (AM) qui vont subir une transition mésenchyme-épithélium à l'origine de la formation d'une vésicule rénale (VR) épithéliale. Par des processus de régionalisation et différenciation regroupés sous le terme de néphrogenèse, cette VR va aboutir à la formation d'un « corps en $S$ » (SSB), puis d'un néphron régionalisé constitué d'un glomérule (G) en relation avec l'endothélium vasculaire (en rouge), un tubule proximal (TP), une anse de Henlé (AH) et un tubule distal (TD) qui va se connecter au réseau de canaux collecteurs (CC). La signalisation Wntll canonique est requise lors de l'interaction des cellules des extrémités des branches du BU pour promouvoir le branchement via la cascade Ret-Wnt11-GDNF (glial cell-derived neurotrophic factor), définissant ainsi le nombre et la complexité de l'arborescence des canaux collecteurs. Le branchement est donc dépendant de la voie Wnt canonique et de la $\beta$-caténine qui maintiennent un état indifférencié de l'extrémité des branches. En parallèle, un signal Wnt9b provenant de l'épithélium du BU va conduire à activer la voie canonique déclenchant le branchement précocement puis, lors de l'induction de la néphrogenèse, agit sur la population de MM, permettant son agrégation en AM puis la transition épithéliomésenchymateuse, contrôlant ainsi la néphrogenèse par l'activation d'une cascade dépendante de Fgf8 (fibroblast growth factor 8)-Wnt4. Wnt9b est également capable de promouvoir, lors de la morphogenèse, l'élongation des tubules par les processus de convergence-extension dépendants de la voie Wnt/PCP non canonique [48]. (c) Maria Kuzmakuzniarska, website:http://mybioscience.org.

[27]. Ainsi, la perte de fonction des gènes $P k d l$ ou Kif3a au cours du développement conduit à la formation rapide de kystes et à une insuffisance rénale, alors que ceux-ci se développent beaucoup plus lentement si l'inactivation génique n'est induite qu'au-delà du jour 13 postnatal $[23,25,26,28]$. L'altération de la prolifération ne semble pas être seule en cause dans la formation des kystes. La sévérité de la pathologie est en relation avec l'état de différenciation et de maturation terminale du rein [27]. Elle peut aussi varier selon le seuil de protéines fonctionnelles présentes, indiquant un effet de dosage génique [29]. Ces résultats indiquent que plusieurs mécanismes cellulaires distincts contrôlent l'initiation et la progression des kystes rénaux au cours du développement embryonnaire ou chez l'adulte, mécanismes actuellement en cours d'étude.

\section{Fonction du cil dans les tubules rénaux}

Les cils primaires sont présents à la surface de presque tous les épithéliums du rein, qu'ils dérivent du bourgeon urétéral (BU) ou des cellules mésenchymateuses (MM). Ils sont en contact avec la lumière des tubules du néphron et des canaux collecteurs. Si leur rôle au cours du développement rénal reste encore mal caractérisé, des travaux récents portant notamment sur KIF3a montrent leur importance dans l'arborescence et la néphrogenèse, respectivement via le contrôle de la prolifération ou de l'apoptose [30]. Dans les cellules rénales différenciées, les cils semblent intervenir comme mécanosenseurs du flux, une fonction importante pour le maintien de la fonction rénale. En réponse au flux d'urine, la flexion du cil déclencherait une augmentation transitoire du calcium intracellulaire, provenant du réticulum endoplasmique et du compartiment extracellulaire ; cette réponse requiert l'intégrité du cil, au moins dans les cellules en culture [31, 32]. Ce rôle de senseur de la pression du flux dépend du taux respectif des protéines $\mathrm{PCl}$ et PC2 $[4,5,33]$. Ainsi, chez les souris $P k d l^{-/}$, le cil des cellules des tubules proximaux se forme normalement, mais la réponse calcique qu'il induit est défectueuse [34]. La perte ou le dysfonctionnement du cil bloqueraient donc 
la signalisation calcique, déclenchant la progression du cycle cellulaire et la sécrétion de fluide, deux processus potentiellement impliqués dans la progression des kystes.

La stimulation mécanique du cil par le flux module un nombre important d'effecteurs de voies de signalisation impliquées dans le contrôle du cycle cellulaire, notamment celles qui font intervenir l'AMPc, JAK (Janus kinase)/STAT (signal transducer and activator of transcription) et mTOR (mammalian target of rapamycin) [31]. Celles-ci sont globalement affectées chez les patients atteints de polykystose de type ADPKD et ARPKD. In vitro, la perte du cil dans des cellules $\mathrm{Kif3}^{-/-}$est suffisante pour augmenter le niveau d'AMPc intracellulaire [35], et in vivo, le blocage de la signalisation calcique chez les souris mutantes pour PC2 augmente le niveau d'AMPc dans les kystes et active la voie de signalisation en aval. De plus, dans différents modèles kystiques associés à une surexpression du récepteur V2 de la vasopressine, le blocage pharmacologique de ce récepteur diminue le taux d'AMPc et réduit la progression des kystes [36]. Le flux pourrait, par ailleurs, déclencher un signal de maintien de la différenciation cellulaire et un blocage de la croissance/prolifération via la voie JAK/STAT [31].

Enfin, il existe un lien entre la voie de signalisation mTOR et le cil, notamment dans le contrôle de la traduction, de la croissance et de la prolifération cellulaires [37]. Cette voie est hyperactivée dans les épithéliums kystiques des PKD et des $\mathrm{NHPH}$, et le traitement par la rapamycine (un inhibiteur de $\mathrm{mTOR}$ ) réduit la formation des kystes dans ces modèles [38]. La perte du cil, ou de sa fonction de senseur de flux, conduirait à une dérégulation d'un complexe $\mathrm{PCl} / \mathrm{mTOR}$, provoquant une augmentation de la prolifération et de la croissance cellulaires et la formation de kystes, mais indépendamment de la signalisation calcique [39]. Toutefois, des travaux très récents analysant la relation génétique entre polycystines et suppression du cil chez les souris PKD, suggèrent que les voies MAPK (mitogen activated protein kinase)/ERK (extracellular signal-regulated kinase), mTOR et de I'AMPc ne sont que très peu susceptibles d'être à l'origine de signaux de croissance des kystes dépendants du cil [40]. Les auteurs proposent plutôt l'existence de voies divergentes, soit dépendantes des polycystines, soit affectées par la perte du cil. Chez l'adulte, ces nouvelles voies de formation de kystes contrôlées par le cil seraient supprimées en présence des polycystines fonctionnelles. De même, d'autres travaux, se fondant sur des modèles de lésion du rein chez l'adulte, suggèrent que les altérations de la prolifération ne peuvent pas être seules en cause dans la genèse des kystes chez les souris mutantes pour Ift88 ou Kif3a, dont le cil est altéré, et un rôle important des processus d'acquisition de la polarité cellulaire a été en effet proposé [26, 41].

\section{Kystes et polarité cellulaire}

Le lien dans le rein entre kyste et polarité cellulaire est maintenant bien documenté, en partie en raison de la localisation extraciliaire des protéines PCl et - 2 ou NPHP au niveau des jonctions cellulaires, ou de leur implication dans l'adhérence des cellules à la matrice extracellulaire (MEC) $[72](\rightarrow)$.

$(\rightarrow)$ Voir la Synthèse de N. Taulet et B. Delaval, page 1040 de ce numéro
En effet, dans le rein des patients NPHP ou ADPKD/ ARPKD, on observe des défauts d'organisation de la lame basale et des jonctions serrées. L'absence de protéine impliquée dans la formation et la stabilité du centrosome, par exemple en présence d'une mutation nulle du gène codant pour la galectine 3 [42], ou même celle de NPHPI/-3/-4/-6/-8 et IFT88, provoquent des défauts de polarisation apico-basale des cellules épithéliales du rein adulte. Des expériences de ciliogenèse et de morphogenèse épithéliale en culture 3D de cellules rénales suggèrent des défauts de polarité, principalement d'organisation apicale; ceux-ci sont associés à la formation de structures épithéliales à lumières multiples $[8,9,42,43]$. Ces données suggèrent, entre autres, un rôle de ces protéines dans la sécrétion et l'assemblage de la matrice extracellulaire, et le dysfonctionnement de ce processus pourrait être une cause de formation de kystes dans le rein [44]. De plus, in vitro, $\mathrm{PCl}$ régule l'organisation du cytosquelette d'actine lors de la migration directionnelle de cellules embryonnaires de reins [45], et ce cytosquelette est également désorganisé in vivo chez les patients atteints de polykystose (ADPKD).

Polarité apico-basale, mise en place de la matrice extracellulaire et orientation du cytosquelette peuvent être le résultat de l'établissement de la polarité planaire cellulaire (planar cell polarity, PCP) [73] $(\rightarrow)$.

$(\rightarrow)$ Voir la Synthèse

de J. Ezan et

M. Montcouquiol, page 1004 de ce numéro

Le lien entre formation des kystes et établissement de la polarité planaire cellulaire est bien documenté, principalement par la fonction de cette dernière dans les processus d'orientation du fuseau de division (aussi nommé oriented cell division, $O C D$ ) et de convergenceextension $(C / \varepsilon)$.

\section{Rôle de l'orientation du fuseau mitotique}

Depuis la publication de défauts de polarité planaire et d'orientation du fuseau mitotique dans les tubules de rat $P C K$, modèles des ARPKD par atteinte de Pkhdl [46], de nombreuses équipes se sont intéressées aux processus d'orientation du fuseau mitotique - et donc de division cellulaire - dans l'apparition des kystes rénaux (Figure 2). En effet, les cellules mitotiques orientent préférentiellement leur fuseau parallèlement à l'axe du tube, générant ainsi deux cellules filles qui se superposent et permettent l'allongement du tubule sans perturber son diamètre. Le rôle du cil dans le contrôle

\footnotetext{
${ }^{1}$ La polarité planaire désigne le processus par lequel toutes les cellules d'un épithélium deviennent polarisées et prennent la même orientation dans le plan du tissu, en réponse à différentes voies de signalisation.
} 
de cette orientation dans le rein reste actuellement controversé. Même si IFT88 est directement impliquée dans ce processus, les résultats diffèrent selon les IFT étudiées [17, 18]. Le rôle du cil dans la régulation des voies de polarité planaire pourrait passer par le contrôle de la maturation et de la position du centrosome dans l'établissement du fuseau mitotique [47]. Si ce processus d'orientation du fuseau mitotique peut expliquer l'origine des kystes qui apparaissent après la naissance (à partir du jour 14) chez la souris, il n'est pas responsable des kystes qui apparaissent lors du développement embryonnaire, car, à cette période, les cellules se divisent dans des directions aléatoires. En effet, le fuseau mitotique ne s'oriente dans l'axe du tubule qu'à partir du $5^{\mathrm{e}}$ jour postnatal [48]. Enfin, des données indiquent que les défauts d'orientation du fuseau mitotique ne préexisteraient pas à l'apparition des kystes chez les mutants $P k d l$ et $P k d 2$, mais en seraient la conséquence. De plus, les défauts d'orientation du fuseau mitotique observés chez les mutants $P k h d l^{\text {del4/del } 4}$ ne seraient pas suffisants pour entraîner le développement de kystes dans ce modèle [49].

\section{Rôle dans la convergence-extension}

La voie de la polarité planaire semble affectée lors de la formation des kystes via son rôle dans le contrôle des mouvements de convergenceextension $(C / \varepsilon)$, qui gouvernent l'allongement du tubule lors de la morphogenèse ou lors de la réparation d'erreurs apparues lors d'orientation du fuseau mitotique (Figure 3 ). Les mouvements de convergence-extension, qui remodèlent un tubule en diminuant son diamètre et allongeant son axe, résultent de l'intercalation des cellules polarisées et allongées de l'épithélium perpendiculairement à l'axe du tube. Le maintien d'un trop grand nombre de cellules dans le diamètre des tubules, comme chez le mutant hypomorphe Wnt9b, affecte précocement les fonctions de réabsorption des cellules tubulaires, et l'augmentation de la pression hydrostatique participe à la formation d'un kyste [48]. La visualisation des réarrangements cellulaires au cours de l'allongement du tubule rénal de xénope vient renforcer ce modèle de convergence-extension, mécanisme sous-tendu par la modification dynamique du cytosquelette (actine-myosine) [50]. En revanche, l'allongement du tubule rénal dans le rein du poisson zèbre dépend, lui, de mouvements de migration collective qui activeraient un signal de prolifération et seraient directement dépendants du flux de liquide [51]. Plus récemment, le rôle central de PCl dans l'acquisition de la polarité cellulaire dans les cellules des canaux collecteurs a été décrit comme essentiel aux mouvements de migration orientée et de convergence-extension, et cela via son interaction avec les complexes de polarité Par3/aPKC (atypical protein kinase C) au cours du développement [52].

\section{Cils et voies de signalisation impliquées dans le développement embryonnaire du rein}

Le lien entre le cil et l'établissement de la polarité cellulaire est suggéré par le fait que le cil et/ou le centrosome jouent un rôle de plate-forme d'intégration de plusieurs voies de signalisation contrôlant la prolifération cellulaire, telles que les voies Wnt et Hedgehog ( $\mathrm{Hh})$, également impliquées dans le développement embryonnaire. La dérégulation de ces

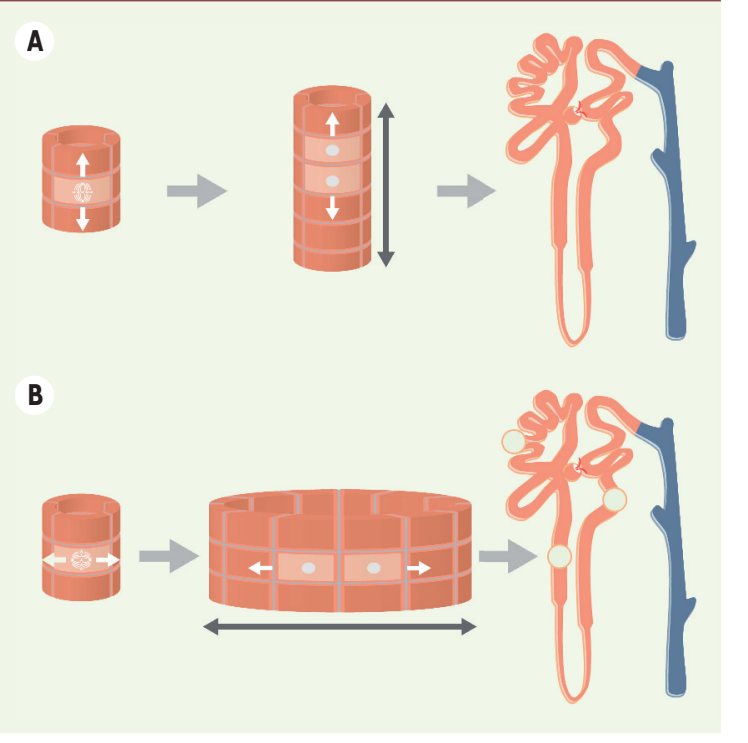

Figure 2. Rôle de l'orientation du fuseau mitotique. A. Au cours de l'élongation normale des tubules rénaux, il a été décrit que les cellules mitotiques orientent préférentiellement leur fuseau parallèlement à l'axe du tube, générant ainsi deux cellules filles qui se superposent et permettent l'allongement du tubule sans perturber son diamètre (double flèche). B. La perte du contrôle de l'orientation de division conduit à ce que le fuseau s'établisse de manière aléatoire et génère alors deux cellules filles pouvant s'orienter perpendiculairement à l'axe du tubule, conduisant à un élargissement de son diamètre (double flèche), phénomène qui pourrait être à l'origine des kystes (dilatations vert clair). (c) Maria Kuzmakuzniarska, website:http://mybioscience.org.

voies, pourtant régulées séparément, au niveau de l'axonème pour la voie th et au niveau du corps basal pour les voies Wnt canonique et polarité planaire, pourrait être à l'origine des processus moléculaires responsables de l'initiation de la formation de kystes [53].

\section{Voie Hh dans le rein}

Le rôle de la voie Hh au cours du développement rénal est peu documenté. L'activité de cette voie semble restreinte à la région médullaire du rein et à l'uretère. Les mutants Sonic hedgehog (Shh) ne développent pas de kystes rénaux, et l'activation ectopique de Shh dans la région corticale du rein provoque des réductions importantes de la néphrogenèse et de l'arborescence, corrigées par l'expression de la forme constitutivement active du répresseur Gli3 (Gli3R) $[54,74](\rightarrow)$.

$(\rightarrow)$ Voir la Synthèse de C. Laclef, page 980 de ce numéro

Des données récentes ont permis de montrer, pour la première fois, le rôle du cil dans le contrôle de la prolifération du bourgeon urétéral et de l'apoptose du mésenchyme rénal lors de la néphrogenèse. La question du lien 
entre Shh et cil dans le rein est longtemps restée en suspens puisque les mutants Shh ne développent pas de kystes. Mais ce travail montre que Shh et cil sont tous deux importants pour le développement normal du rein, chacun des quatre types de données développés ci-dessous (concernant les mutants Kif3A-Gli3R ; Thml-Iftl40; Mksl ; Glis2) apportant des arguments en ce sens. Les mutants conditionnels Kif3a, dont les dérivés du bourgeon urétéral sont dépourvus de cils, se caractérisent par des réductions de l'arborescence que corrige l'expression de la forme Gli3R constitutive, soulignant le rôle du cil dans la signalisation Hh également dans le rein [30]. De plus, les délétions de Thm1 (TPR-containing Hedgehog modulator 1)-Ift 139 et Ift 140 altèrent la formation des cils au cours de l'embryogenèse chez la souris, provoquent des kystes rénaux et s'accompagnent d'une augmentation des transcrits Gli et de la signalisation Hh, faisant le lien entre voies Hh, cils et kystes rénaux [18, 55]. Les souris mutantes $M k s$ l présentent, outre des kystes glomérulaires et tubulaires congénitaux, des anomalies urétérales qui pourraient résulter d'un dysfonctionnement de la voie Shh [10]. Enfin, le gène Glis2, affecté dans les ciliopathies NPHP7, code pour un facteur de transcription à doigts de zinc analogue aux Gli, qui semble important pour la répression de Hh dans le rein en période postnatale. Son altération pourrait faire le lien entre les NPHP et un éventuel maintien de l'activité des voies Hh et Wnt canoniques dans le rein [56]. L'induction de kystes rénaux par les glucocorticoïdes pendant la gestation impliquerait également une voie hedgehog, via une surexpression d'indian Hh (Ihh) qui serait un stimulus important de la progression des kystes [57]. L'augmentation de la signalisation Hh au cours du développement embryonnaire pourrait être un signal important pour le développement de kystes rénaux.

\section{Voie Wnt dans le rein}

De nombreuses études décrivent l'implication de la voie Wnt au cours du développement du rein (Figure 1) [20]. Le cil primaire aurait un rôle important dans la régulation de la balance entre les voies Wnt canonique/ $\beta$-caténine et Wnt/PCP via la fonction de I'INV/NPHP2 [58]. Dans de nombreux tissus, l'absence de cil provoque une suractivation de la voie Wnt canonique, suggérant un rôle répresseur du cil dans la régulation de cette voie. Si ce rôle est démontré dans le rein du poisson zèbre et du xénope [59], ce n'est pas aussi clair dans le rein de souris. II n'y a aucune altération de la signalisation Wnt canonique [60] chez les mutants Inv de souris, et l'activité de cette voie ne semble pas liée à l'apparition des kystes dans des modèles murins (mutants $P k d l$ et $P k d 2$ ) congénitaux et adultes de ADPKD [6l].

II semblerait que ce soit plutôt le passage vers la voie Wnt/PCP2 qui soit altéré lors de l'apparition de kystes au cours du développement [73] $(\rightarrow)$. En effet, les mutants Wnt9b de souris, inca- $\rightarrow$ Voir la Synthèse de J. Ezan et M. Montcouquiol, page 1004 de ce numéro pables de promouvoir la voie de la polarité planaire cellulaire lors de l'élongation des tubules par les processus de convergence-extension, développent des kystes congénitaux dès ع15,5 [48] (Figure 3). Les

\footnotetext{
${ }^{2}$ La voie Wnt canonique et PCP mettent en jeu un certain nombre d'intervenants communs qui semblent activer l'une ou l'autre des voies via le cil et permettent une balance entre ces deux voies, conférant au cil un rôle d'interrupteur.
}

protéines associées au cil sont nécessaires à l'activation de la voie Wnt-PCP. Dans le rein du poisson zèbre, il a été montré que les produits des gènes Nphp4 et Inv/Nphp2 régulent la localisation et la quantité de la protéine cytoplasmique Dishevelled (Dvl) - une des protéines des voies Wnt canonique et PCP - et activent, selon la dose, la voie Wnt canonique ou celle de la polarité planaire cellulaire. Ainsi, des dérégulations de Nphp4 et Dvl aboutissent à la formation de kystes associés à des défauts d'élongation et de réarrangements cellulaires [62]. En parallèle, les souris porteuses d'une mutation de Vangl2 (van Gogh-like protein 2), un gène de la voie pola- $(\rightarrow)$ Voir la Synthèse de J. Ezan et M. Montcouquiol, page 1004 de ce numéro arborent des défauts dans l'arborescence du bourgeon urétéral du rein, suggérant que cette voie peut également être impliquée [63].

\section{Voies de polarité planaire dans le rein}

Il convient également de mentionner l'implication d'autres voies de polarité planaire régulant la polarisation du cytosquelette, la forme de la cellule et l'orientation du fuseau mitotique dans le rein, telles que la voie des cadhérines Fat/Dachsous (Ds), également associée à la formation de kystes rénaux au cours du développement embryonnaire. Ainsi, les souris $\mathrm{Fat}^{-/-}$ ont des reins kystiques associés à des défauts d'arborescence du bourgeon urétéral et de l'orientation du fuseau mitotique; ces formations kystiques sont augmentées par les autres mutants de la voie (Fatl, Dchs l) [64]. L'action des Fat semble passer par la régulation de la voie Hippo (impliquée dans la croissance des tissus), au moins dans le pronéphros du poisson zèbre [69]. Cette voie Hippo interviendrait aussi dans les processus de tubulogenèse au cours de la néphrogenèse. Enfin, il a été récemment montré que les voies Fat-PCP et Wnt-PCP pourraient agir en synergie dans le rein de mammifère, puisque la perte de Vangl2 aggrave les phénotypes kystiques des mutants Fat4 [65].

\section{Conclusions et perspectives}

L'existence d'un lien causal entre cils et kystes rénaux reste encore aujourd'hui une question non résolue; cela s'explique par les nombreuses fonctions extraciliaires des protéines qui se localisent au niveau du cil [72] $(\rightarrow)$, et la variabilité des tissus $\rightarrow$ Voir la Synthèse de N. Taupet et B. Delaval, page 1040 de ce numéro affectés par la formation des kystes. Si IFT88, IFT20 ou KIF3a sont importantes pour la structure, la longueur et la morphologie du cil, c'est moins clair pour la fibrocystine. 


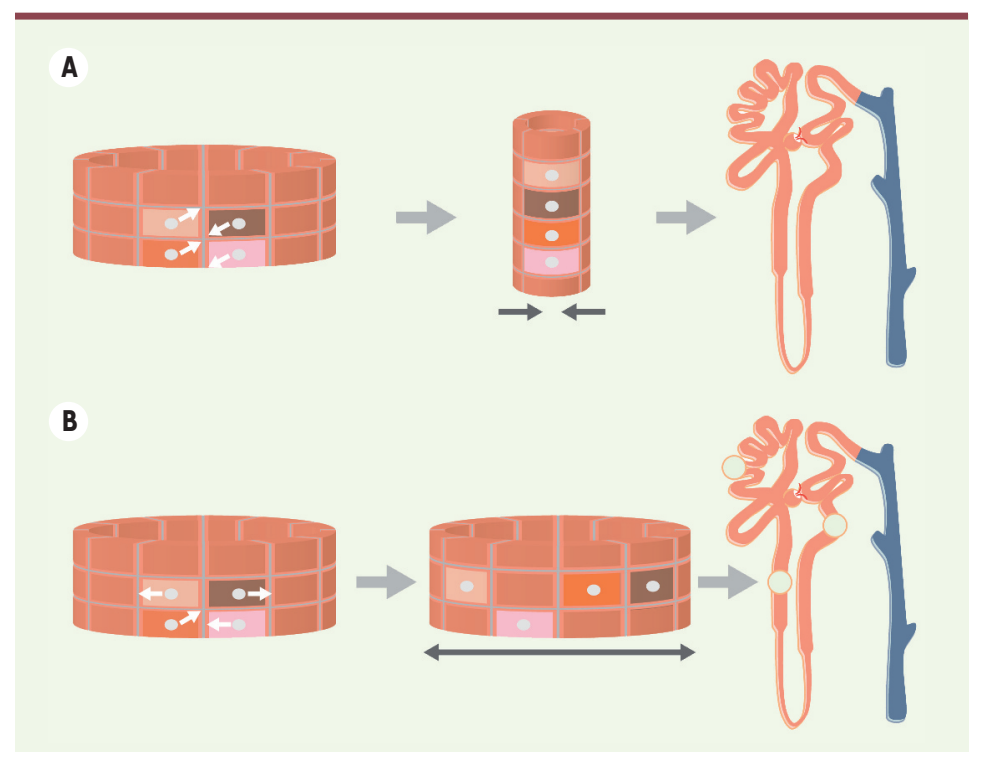

Figure 3. Rôle dans la convergence/extension. A. Pendant le processus de convergence/extension, les cellules acquièrent une polarité médio-latérale et subissent des mouvements d'intercalation perpendiculairement à l'axe du tube (flèches blanches), conduisant à diminuer son diamètre et à allonger sa taille (flèches noires têtes bèches). $B$. Si la polarité des cellules se fait de manière aléatoire, leurs intercalations ne sont pas coordonnées (flèches blanches), ce qui conduit à maintenir un trop grand nombre de cellules dans le diamètre du tubule dont la morphogenèse est alors altérée (double flèche noire). La dilatation locale provoque l'apparition de kystes (dilatations vert clair). (c) Maria Kuzmakuzniarska, website:http:// mybioscience.org.

mécanismes de kystogenèse, étudier le lien existant avec les altérations du métabolisme [67], et comprendre le rôle que peuvent y jouer les microARN,

Même si la morphologie des cils est intacte chez les mutants perte de fonction des $\mathrm{PCl} / 2$, de la cystine ou de l'inversine, il faut être prudent, au vu des autres localisations cellulaires de ces protéines, avant d'attribuer à l'altération de la fonction ciliaire la responsabilité des altérations de la prolifération et de la réponse calcique observées chez les mutants PKD. L'un des principaux défis est donc de déterminer quelles fonctions cellulaires sont associées à ces protéines, qu'elles soient localisées au niveau ciliaire ou pas. D'autres études visent à distinguer les aspects fonction et formation du cil, afin d'identifier leur impact respectif dans l'émergence des kystes. Ainsi, des souris mutantes $\operatorname{Tg} 737^{\text {Polaris }}$ chez lesquelles un transgène Ift88 est surexprimé, développent encore des kystes rénaux à la naissance, malgré la morphologie normale des cils en microscopie électronique dans les épithéliums kystiques et non kystiques [66]. À l'inverse, la perte de fonction de KIF3a précocement au cours du développement du rein - qui conduit à une disparition graduelle des cils dès $\varepsilon 13,5$ chez la souris - ne provoque pas l'apparition de kystes au cours du développement embryonnaire, mais seulement après la naissance. Ces observations suggèrent, là encore, que les cils n'ont pas de responsabilité directe dans la formation des kystes congénitaux [30]. Enfin, et de façon inattendue, il semblerait que des cils intacts soient nécessaires à la croissance rapide des kystes après la naissance dans des modèles murins de PKD [40]. En effet, la suppression des cils par l'induction de mutations Kif3A et Ift20 abolit la croissance rapide des kystes chez les souris inactivées pour $P C 1$ ou $P C 2$, indiquant que les cils intacts fournissent un signal positif pour la croissance du kyste, signal réprimé par la fonction normale des polycystines.

À l'avenir, il faudrait donc différencier les mécanismes de croissance rapide des kystes au cours du développement embryonnaire ou chez le jeune, de ceux impliqués dans la croissance lente chez l'adulte ou rapide après un signal de lésion-régénération. De même, il faudra réussir à découpler les processus de formation des kystes selon qu'ils affectent les fonctions cellulaires mécanosensorielles, prolifératives, de polarité ou les voies de signalisation développementales. Enfin, il faudra poursuivre l'analyse des réseaux de gènes impliqués dans les puisque de nombreuses études montrent leur implication, comme la perte de fonction de Dicer dans le rein [68]. Ces complexités nous confrontent à de nouveaux défis, notamment pour orienter les études des différentes thérapies qui aboutiront à la réduction de la progression des kystes rénaux, voire à leur complète résorption. $\diamond$

\section{SUMMARY}

Cilia and renal cysts

Advances in genomics, bioinformatics and the creation of model organisms have identified many genes associated with polycystic kidney diseases. Historically, these genes were not necessarily associated with ciliopathies, but it appeared that many connections can be made between the cystic kidney disease and function of the primary cilium. Indeed, the proteins encoded by these genes are localized to the cilium itself, to the basal body or are known to regulate the expression and localization of ciliary proteins. The goal of this article is to describe the multiple cellular processes that may lead to the development of renal cysts if they are deregulated. These include changes in proliferation rate, cell polarity or signaling pathways involved in embryonic kidney development. To highlight the role of the primary cilium in cystogenesis, I will discuss several studies investigating the function of ciliary genes and cilia in the kidneys of different model organisms. $\diamond$

\section{LIENS D'INTÉRÊT}

L'auteur déclare n'avoir aucun lien d'intérêt concernant les données publiées dans cet article. 


\section{RÉFÉRENCES}

1. Pan J, Seeger-Nukpezah T, Golemis EA. The role of the cilium in normal and abnormal cell cycles: emphasis on renal cystic pathologies. Cell Mol Life Sci $2013 ; 70: 1849-74$.

2. Hildebrandt F, Benzing T, Katsanis N. Ciliopathies. N Engl J Med 2011 ; 364 : 1533-43.

3. Gallagher AR, Germino GG, Somlo S. Molecular advances in autosomal dominant polycystic kidney disease. Adv Chronic Kidney Dis 2010 ; 17 : 118-30.

4. Nickel C, Benzing T, Sellin L, et al. The polycystin-1 C-terminal fragment triggers branching morphogenesis and migration of tubular kidney epithelial cells. J Clin Invest 2002 ; 109 : 481-9.

5. Streets AJ, Wessely 0 , Peters DJ, Ong AC. Hyperphosphorylation of polycystin-2 at a critical residue in disease reveals an essential role for polycystin-1-regulated dephosphorylation. Hum Mol Genet $2013 ; 22$ : 1924-39.

6. Watnick TJ, Germino GG. Polycystic kidney disease: Polycystin-1 and polycystin-2-it's complicated. Nat Rev Nephrol $2013 ; 9: 249-50$.

7. Wu Y, Dai XQ, Li $Q$, et al. Kinesin-2 mediates physical and functional interactions between polycystin-2 and fibrocystin. Hum Mol Genet 2006 ; 15 : 3280-92.

8. Delous M, Hellman NE, Gaude HM, et al. Nephrocystin-1 and nephrocystin-4 are required for epithelial morphogenesis and associate with PALS1/PATJ and Par6. Hum Mol Genet 2009 ; 18 : 4711-23.

9. Ghosh AK, Hurd T, Hildebrandt F. 3D spheroid defects in NPHP knockdown cells are rescued by the somatostatin receptor agonist octreotide. Am J Physiol Renal Physiol 2012 ; 303 : F1225-9.

10. Cui C, Chatterjee B, Francis D, et al. Disruption of Mksl localization to the mother centriole causes cilia defects and developmental malformations in Meckel-Gruber syndrome. Dis Model Mech $2011 ; 4: 43-56$.

11. Phillips CL, Miller KJ, Filson AJ, et al. Renal cysts of inv/inv mice resemble early infantile nephronophthisis. J Am Soc Nephrol 2004 ; 15 : 1744-55.

12. Shiba D, Takamatsu T, Yokoyama T. Primary cilia of inv/inv mouse renal epithelial cells sense physiological fluid flow: bending of primary cilia and $\mathrm{Ca}^{2+}$ influx. Cell Struct Funct $2005 ; 30$ : 93-100.

13. Yoder BK, Hou X, Guay-Woodford LM. The polycystic kidney disease proteins, polycystin-1, polycystin-2, polaris, and cystin, are co-localized in renal cilia. J Am Soc Nephrol 2002 ; 13 : 2508-16.

14. Pazour GJ, Dickert BL, Vucica Y, et al. Chlamydomonas IFT88 and its mouse homologue, polycystic kidney disease gene tg737, are required for assembly of cilia and flagella. J Cell Biol $2000 ; 151$ : 709-18.

15. Halbritter J, Bizet AA, Schmidts M, et al. Defects in the IFT-B component IFT172 cause Jeune and Mainzer-Saldino syndromes in humans. Am J Hum Genet $2013 ; 93: 915-25$.

16. Schmidts M, Frank V, Eisenberger T, et al. Combined NGS approaches identify mutations in the intraflagellar transport gene IFT140 in skeletal ciliopathies with early progressive kidney disease. Hum Mutat $2013 ; 34: 714-24$

17. Jonassen JA, SanAgustin J, Follit JA, Pazour GJ. Deletion of IFT20 in the mouse kidney causes misorientation of the mitotic spindle and cystic kidney disease.J Cell Biol $2008 ; 183: 377-84$

18. Jonassen JA, SanAgustin J, Baker SP, Pazour GJ. Disruption of IFT complex A causes cystic kidneys without mitotic spindle misorientation. J Am Soc Nephrol $2012 ; 23: 641-51$.

19. Cong $\varepsilon H$, Bizet $A A$, Boyer 0 , et al. A Homozygous missense mutation in the ciliary gene TTC21B causes familial FSGS. J Am Soc Nephrol 2014 ; Doi : 10.1681.

20. Costantini F. Genetic controls and cellular behaviors in branching morphogenesis of the renal collecting system. Wiley Interdisc Rev Dev Biol 2012 ; 1 : 693-713.

21. Olteanu D, Liu X, Liu W, et al. Increased $\mathrm{Na}^{+} / \mathrm{H}^{+}$exchanger activity on the apical surface of a cilium-deficient cortical collecting duct principal cell model of polycystic kidney disease. Am J Physiol 2006 ; 302 : C1436-51.

22. Lu W, Peissel B, Babakhanlou $H$, et al. Perinatal lethality with kidney and pancreas defects in mice with a targetted Pkdl mutation. Nat Genet 1997 ; 17 : 179-81.

23. Piontek K, Menezes LF, Garcia-Gonzalez MA, et al. A critical developmental switch defines the kinetics of kidney cyst formation after loss of Pkdl. Nat Med $2007 ; 13: 1490-5$.

24. Lantinga-van Leeuwen IS, Leonhard WN, van der Wal A, et al. Kidney-specific inactivation of the Pkdl gene induces rapid cyst formation in developing kidneys and a slow onset of disease in adult mice. Hum Mol Genet 2007 ; 16 : 3188-96.

25. Davenport JR, Watts AJ, Roper VC, et al. Disruption of intraflagellar transport in adult mice leads to obesity and slow-onset cystic kidney disease. Curr Biol 2007; $17: 1586-94$.

26. Patel V, Li L, Cobo-Stark P, et al. Acute kidney injury and aberrant planar cell polarity induce cyst formation in mice lacking renal cilia. Hum Mol Genet 2008 ; 17 : 1578-90.

27. Menezes LF, Germino GG. Polycystic kidney disease, cilia, and planar polarity. Methods Cell Biol $2009 ; 94: 273-97$

28. Lin F, Hiesberger T, Cordes K, et al. Kidney-specific inactivation of the KIF3A subunit of kinesin-II inhibits renal ciliogenesis and produces polycystic kidney disease. Proc Natl Acad Sci USA 2003 ; $100: 5286-91$.

29. Hopp K, Ward CJ, Hommerding CJ, et al. Functional polycystin-1 dosage governs autosomal dominant polycystic kidney disease severity. J Clin Invest 2012 ; 122 : 4257-73.

30. Chi L, Galtseva A, Chen L, et al. Kif3a controls murine nephron number via GLI3 repressor, cell survival, and gene expression in a lineage-specific manner. PloS One 2013; 8 : e65448.

31. Zhou J. Polycystins and primary cilia: primers for cell cycle progression. Annu Rev Physiol 2009; $71: 83-113$.
32. Patel A. The Primary cilium calcium channels and their role in flow sensing. Pflugers Arch 2014 ; Doi : 10.1007/s00424-014-1516-0.

33. Sharif-Naeini R, Folgering JH, Bichet $D$, et al. Polycystin-1 and -2 dosage regulates pressure sensing. Cell 2009 ; $139: 587-96$.

34. Nauli SM, Alenghat FJ, Luo $Y$, et al. Polycystins 1 and 2 mediate mechanosensation in the primary cilium of kidney cells. Nat Genet $2003 ; 33$ : 129-37.

35. Choi $\mathrm{YH}$, Suzuki A, Hajarnis S, et al. Polycystin-2 and phosphodiesterase $4 \mathrm{C}$ are components of a ciliary A-kinase anchoring protein complex that is disrupted in cystic kidney diseases. Proc Natl Acad Sci USA 2011 ; 108 : 10679-84.

36. Torres VE, Harris PC. Strategies targeting cAMP signaling in the treatment of polycystic kidney disease. J Am Soc Nephrol 2014 ; 25 : 18-32.

37. Boletta $A$. Emerging evidence of a link between the polycystins and the mTOR pathways. PathoGenetics $2009 ; 2: 6$.

38. Gattone VH 2nd, Sinders RM, Hornberger TA, Robling AG. Late progression of renal pathology and cyst enlargement is reduced by rapamycin in a mouse model of nephronophthisis. Kidney Int 2009 ; 76 : 178-82.

39. Boehlke C, Kotsis F, Patel V, et al. Primary cilia regulate mTORCl activity and cell size through Lkbl. Nat Cell Biol $2010 ; 12$ : 1115-22.

40. Ma M, Tian X, Igarashi P, et al. Loss of cilia suppresses cyst growth in genetic models of autosomal dominant polycystic kidney disease. Nat Genet 2013; 45 : 1004-12.

41. Sharma N, Malarkey $\varepsilon B$, Berbari NF, et al. Proximal tubule proliferation is insufficient to induce rapid cyst formation after cilia disruption. J Am Soc Nephrol $2013 ; 24: 456-64$.

42. Koch A, Poirier F, Jacob R, Delacour D. Galectin-3, a novel centrosomeassociated protein, required for epithelial morphogenesis. Mol Biol Cell $2010 ; 21: 219-31$.

43. Vijayakumar S, Dang S, Marinkovich MP, et al. Aberrant expression of laminin-332 promotes cell proliferation and cyst growth in ARPKD. Am J Physiol Renal Physiol 2014 ; 306 : F640-54.

44. Drummond IA. Polycystins, focal adhesions and extracellular matrix interactions. Biochim Biophys Acta $2011 ; 1812$ : 1322-6.

45. Yao G, Su X, Nguyen V, et al. Polycystin-1 regulates actin cytoskeleton organization and directional cell migration through a novel PCl-pacsin 2-N-wasp complex. Hum Mol Genet 2014 ; 23 : 2769-79.

46. Fischer $\varepsilon$, Legue $\varepsilon$, Doyen $A$, et al. Defective planar cell polarity in polycystic kidney disease. Nat Genet $2006 ; 38: 21-3$.

47. Nigg EA, Stearns T. The centrosome cycle: Centriole biogenesis, duplication and inherent asymmetries. Nat Cell Biol $2011 ; 13: 1154-60$.

48. Karner CM, Chirumamilla R, Aoki S, et al. Wnt9b signaling regulates planar cell polarity and kidney tubule morphogenesis. Nat Genet $2009 ; 41$ : 793-9.

49. Nishio $S$, Tian $X$, Gallagher AR, et al. Loss of oriented cell division does not initiate cyst formation. J Am Soc Nephrol $2010 ; 21: 295-302$.

50. Lienkamp SS, Liu K, Karner CM, et al. Vertebrate kidney tubules elongate using a planar cell polarity-dependent, rosette-based mechanism of convergent extension. Nat Genet $2012 ; 44: 1382-7$.

51. Vasilyev A, Liu Y, Mudumana S, et al. Collective cell migration drives morphogenesis of the kidney nephron. PLoS Biol $2009 ; 7$ : e9.

52. Castelli M, Boca M, Chiaravalli M, et al. Polycystin-1 binds Par3/aPKC and controls convergent extension during renal tubular morphogenesis. Nat Commun 2013; $4: 2658$.

53. Tran PV, Sharma M, Li X, Calvet JP. Developmental signaling: Does it bridge the gap between cilia dysfunction and renal cystogenesis? Birth Defects Res C Embryo Today 2014 ; 102 : 159-73.

54. Cain JE, Islam $\varepsilon$, Haxho F, et al. GLI3 repressor controls nephron number via regulation of Wntll and Ret in ureteric tip cells. PloS One 2009; 4 : e7313.

55. Tran PV, Talbott GC, Turbe-Doan A, et al. Downregulating hedgehog signaling reduces renal ccystogenic potential of mouse models. J Am Soc Nephrol 2014 ; Doi : 10.1681/ASN.2013070735.

56. Li B, Rauhauser AA, Dai J, et al. Increased hedgehog signaling in postnatal kidney results in aberrant activation of nephron developmental programs. Hum Mol Genet $2011 ; 20: 4155-66$.

57. Chan SK, Riley PR, Price KL, et al. Corticosteroid-induced kidney dysmorphogenesis is associated with deregulated expression of known cystogenic molecules, as well as Indian hedgehog. Am J Physiol Renal Physiol 2010 ; 298 : F346-56.

58. Simons M, Gloy J, Ganner A, et al. Inversin, the gene product mutated in nephronophthisis type II, functions as a molecular switch between Wnt signaling pathways. Nat Genet $2005 ; 37: 537-43$.

59. Lancaster MA, Gleeson JG. Cystic kidney disease: the role of Wnt signaling. Trends Mol Med $2010 ; 16: 349-60$. 


\section{RÉFÉRENCES}

60. Sugiyama N, Tsukiyama T, Yamaguchi TP, Yokoyama T. The canonical Wnt signaling pathway is not involved in renal cyst development in the kidneys of inv mutant mice. Kidney Int $2011 ; 79$ 957-65.

61. Miller MM, Iglesias DM, Zhang Z, et al. T-cell factor/beta-catenin activity is suppressed in two different models of autosomal dominant polycystic kidney disease. Kidney Int $2011 ; 80$ : 146-53

62. Burckle C, Gaude HM, Vesque C, et al. Control of the Wnt pathways by nephrocystin- 4 is required for morphogenesis of the zebrafish pronephros. Hum Mol Genet $2011 ; 20: 2611-27$.

63. Yates LL, Papakrivopoulou J, Long DA, et al. The planar cell polarity gene Vangl2 is required for mammalian kidney-branching morphogenesis and glomerular maturation. Hum Mol Genet 2010 ; $19: 4663-76$.

64. Mao Y, Mulvaney J, Zakaria S, et al. Characterization of a Dchs l mutant mouse reveals requirements for Dchs1-Fat4 signaling during mammalian development. Development 2011 ; 138 947-57.

65. Saburi S, Hester I, Goodrich L, McNeill H. Functional interactions between Fat family cadherins in tissue morphogenesis and planar polarity. Development 2012 ; 139 : 1806-20.

66. Brown NE, Murcia NS. Delayed cystogenesis and increased ciliogenesis associated with the re-expression of polaris in Tg737 mutant mice. Kidney Int 2003; $63: 1220-9$.

67. Rowe I, Chiaravalli M, Mannella V, et al. Defective glucose metabolism in polycystic kidney disease identifies a new therapeutic strategy. Nat Med 2014 ; 19 : 488-93.
68. Nagalakshmi VK, Ren $Q$, Pugh MM, et al. Dicer regulates the development of nephrogenic and ureteric compartments in the mammalian kidney. Kidney Int $2011 ; 79: 317-30$.

69. Sharma P, McNeill H. Fat and Dachsous cadherins. Prog Mol Biol Transl Sci $2013 ; 116: 215-35$.

70. Bachmann-Gagescu R. Complexité génétique des ciliopathies et identification de nouveaux gènes. Med Sci (Paris) $2014 ; 30: 1011-23$.

71. Fort $C$, Bastin $P$. Élongation de l'axonème et dynamique du transport intraflagellaire. Med Sci (Paris) $2014 ; 30: 955-61$.

72. Taulet N, Delaval B. De nouvelles fonctions extraciliaires pour les protéines ciliaires. Quelles conséquences sur l'apparition de ciliopathies? Med Sci (Paris) $2014 ; 30: 1040-50$.

73. Ezan J, Montcouquiol M. Les liens multiples entre les cils et la polarité planaire cellulaire. Med Sci (Paris) $2014 ; 30: 1004-10$.

74. Laclef $C$. Le cil primaire, orchestrateur de la morphogenèse cérébrale. Med Sci (Paris) $2014 ; 30: 980-90$

\section{TIRÉS À PART}

M. Paces-Fessy

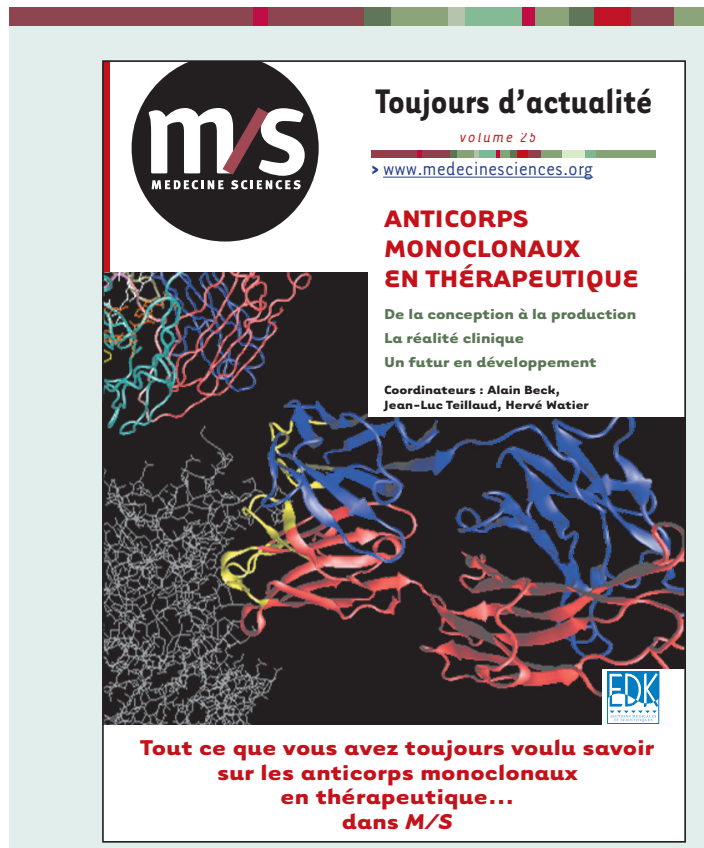

Tout ce que vous avez toujours voulu savoir sur les anticorps monoclonaux en thérapeutique... dans Médecine/Sciences. Pourquoi un numéro spécial de Médecine/Sciences sur les anticorps monoclonaux thérapeutiques? II nous a semblé que le moment était venu de dresser un état des lieux de ces biomédicaments qui prennent désormais une place considérable - et croissante dans les traitements de maladies souvent lourdes et désespérantes. Ce voyage que nous vous proposons à la découverte du monde des anticorps thérapeutiques nous a appris, ou plutôt rappelé, une évidence : les compétences en France sont fortes et nombreuses, qu'elles soient académiques ou industrielles, biotechnologiques ou cliniques. Le paysage français, trop longtemps discret, bruisse désormais de mille initiatives balayant de multiples aspects des anticorps thérapeutiques : études précliniques et cliniques menées avec de nouveaux anticorps dirigés contre des cibles originales, développement de nouveaux formats d'anticorps ou d'anticorps optimisés reposant sur des études structurales et fonctionnelles sophistiquées, recherche active de cibles pertinentes, mise au point de méthodologies de bioproduction, de couplage, etc. L'expansion industrielle rapide de ce champ est un défi que peut et doit relever notre pays, défi tant scientifique qu'économique, avec ses combats pour la propriété intellectuelle et pour l'emploi de nos jeunes scientifiques.

Alain Beck, Jean-Luc Teillaud, Hervé Watier

\section{À retourner à EDK, 109, avenue Aristide Briand, 92541 Montrouge Cedex, France}

Tél. : 0141177405 - Fax : 0149850345 - E-mail : edk@edk.fr

NOM :

Prénom :

Adresse

Code postal :

Ville

Pays :

Fonction :

Je souhaite recevoir $\mathbf{M} / \mathbf{S} \mathbf{n}^{\circ} \mathbf{1 2}$ - décembre 2009 (Anticorps monoclonaux en thérapeutique) : $25 €+3 €$ de port $=\mathbf{2 8} € \mathbf{T T C}$ en exemplaire, soit un total de $€$

$\square$ Par chèque, à l'ordre de $\mathbf{E} \mathbf{D} \mathbf{K}$

Par carte bancaire :

Visa

Eurocard/Mastercard

Carte $n^{\circ}$

Date d'expiration :

$\mathrm{N}^{\circ}$ de contrôle au dos de la carte :

Signature : 